\title{
Service Discovery Framework for Personalized eHealth
}

\author{
Tesfa Tegegne \\ Radboud University \\ Nijmegen, the Netherlands \\ T.Tegegne@cs.ru.nl
}

\author{
Benjamin Kanagwa \\ Makerere University \\ Kampala, Uganda \\ bkanagwa@cit.mak.ac.ug
}

\author{
Theo van der Weide \\ Radboud University \\ Nijmegen, the Netherlands \\ tvdw@cs.ru.nl
}

\begin{abstract}
Personalization of services based on user preferences and user context facilitates service discovery. Several researches have been conducted on service personalization, however, little has been done to allow non-literate users to query services based on their educational level, their linguistic, cognitive, physiological and psychological abilities. In developing countries users have diversified culture, language and traditional values. As a result it is valuable to design the services on the basis of user literacy level, user preference and user context.
\end{abstract}

Keywords-Personalization, User model, Context

\section{INTRODUCTION}

Considering the social, cultural, educational and economic diversity of developing regions, there is a serious challenge to develop appropriate and effective service delivery system that will enable these users to access services that currently remain elusive to them. Adapting an eHealth system to the individual user helps to ensure that the system will be safe and easy to deploy and use as an effective support to individual users. Personalization can thus enhance the users trust in the system, and make it more readily accepted. Personalization can range from simply setting an alarm volume according to the users hearing abilities and the ambient noise level, to the complex tailoring of the users entire eHealth environment. Personalization may even involve language used by the user, for example the personal interpretation of words. Personalization is achieved by maintaining and updating a user profile, which depends on and is dynamically adapted to the users context, general preferences, physical and mental abilities, and other relevant parameters.

Adjusting delivery of healthcare based on patients characteristics, behavior and educational level is beneficial for the patients. eHealth personalization provides opportunities to discover knowledge and services easily according to the user's preferences and context. For example the diabetic patient needs to provide her current sugar level, and report her current weight and blood pressure. The medication she has followed in the past needs to be addressed by the system in order to discover the appropriate treatment and coaching. In our proposed system, first the dialog (communication) is initiated by the user (either a patient or a health professional). The user poses an initial question to the system and the system starts responding and requesting some subsequent inputs: blood pressure, weight, temperature, and the symptoms she has. Based on the given information, the system retrieves available services from the repository. If no service found is found in the repository then the request interpreter will forward for the necessary health provider to address the request manually.

Personalization can be organized using individual or group (stereotype) user modeling. In a stereotype approach, users can be classified into several groups. In eHealth applications users can be classified according to their main diseases, background in medicine (patients, nurses, doctors), general eduction background (no degree, college degree, doctorate, etc), and their tasks (education, consultation, emergency case). Individual patient (user) model, besides the user medical profile, could include their cognitive and psychological behavior, their preferences, and their context. Table 1 indicates an example of a static and dynamic profile of a user (patient).

Related portable devices (cards, chips, tokens, mobile phones, smart devices, sensors, actors, etc.) are considered first line communication tools. They can hold medical / clinical data and improve the communication between patients and health professionals.

The paper is organized as follows: in section 2 personalization on eHealth is discussed. Personal profile is explained in section 3. In section 4 related work is presented. Finally, we conclude the paper and provide future work direction in section 5 .

\section{Personalization on eHealth}

Advances in service orientation, mobile and ubiquitous computing on the one hand, and continuous progress in medical devices and diagnosis methodology on the other hand, are enabling personalized healthcare services to be delivered to individuals at any place and any time [7]. These advances deviate from the One size fits all paradigm in healthcare as is common in traditional hospitals, clinics and healthcare centers. Personalized healthcare ensures that healthcare services provisioned to individuals are customized to their prevailing healthcare needs. In most developed countries, personalized healthcare is provided for citizens through home care, wearable devices, online treatment and 
consultation. In developing countries personalized healthcare could provide an opportunity to compensate for the shortage of professional healthcare workers.

Introducing ICT in the healthcare sector is a hot issue, both in developed and developing countries. Several researchers have been conducting researches how to use ICT in supporting healthcare. Many organizations are involved producing in electronic healthcare standards. For example, Health Level 7 (HL7, [5]) is a health informatics standard organization. The main purpose of this organization is developing, publishing and promoting a comprehensive framework for the development of health informatics standards and the employment of the framework to produce protocol specifications for health data interchange, integration, storage and retrieval among diverse data acquisition, processing, and handling systems.

Similarly, the European Telecommunications Standards Institute (ETSI) is an ICT standard organization. ETSI, recently released a first draft personalization on eHealth standard. This draft standard will help to manage the user profiles for personalization of eHealth systems and services according to the user preferences and needs. This profile is an extension of the 'user profile management (UPM)' standard developed by ETSI see http://portal.etsi.org/stfs/ STF_HomePages/STF352/STF352.asp.

\section{A. eHealth service discovery framework}

Adapting an eHealth system to the individual user is essential for making it safe and easy to deploy and to use as an effective support to independent living. Personalization can thus enhance the user's trust in the eHealth systems, and make them more readily accepted.

The case for personalization of health information is supported by studies in health communication which have shown that health-education material can be much more effective if customized for the individual patient in accordance with their medical condition, demographic variables, personality profile, and other relevant factors [3]. According to [3], little work is being done on what we consider the most important factor: tailoring the information to individual needs, experiences, and communication style.

One of the most important aspects needed in system building is information about the intended users: their goals, needs, moods, preferences, intentions, etc. This information can be acquired through an explicit and implicit process called user modeling. Modeling a user means interpreting user actions within a given system or application. The user model is usually restricted to some characteristics that are supposed to be the most meaningful in the context of the user interaction with the system.

Figure 1 indicates the process of personalized user request.

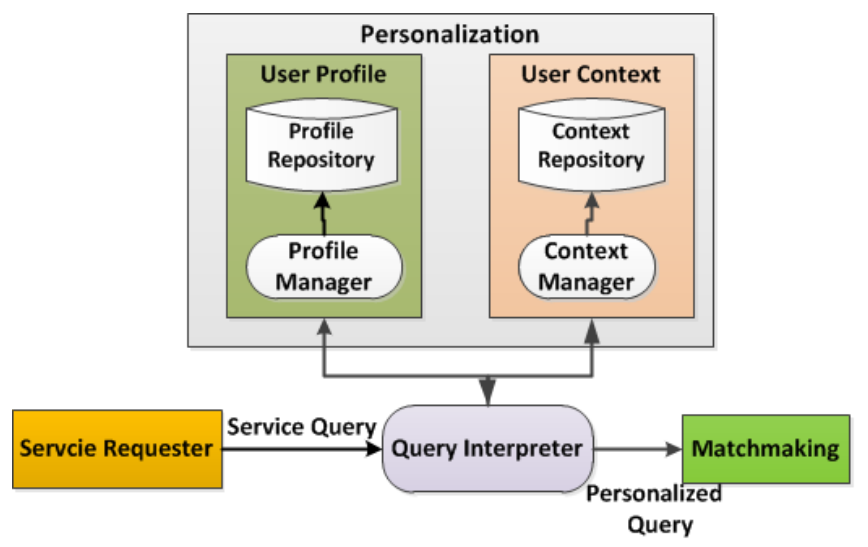

Figure 1. Personalized user request

\section{B. Key benefits}

Personalization in eHealth has twofold advantages; for the health workers it provides information (such as training, professional guidance and assistance) on the basis of their educational background and job descriptions. On the side of the patient, it provides the necessary patient-oriented health information systems like patient preference, informed consent, self-care and shared patient-doctor decision making.

The main aim of personalizing eHealth is to acquire and capture factual information about the patient, their condition, current treatments, their preferences. This information can be obtained from the patient record except the user contextual information.

Personalizing eHealth besides informing, enabling decision making and persuading the patients, it will give users access to medical treatment and consultation services on the basis of their needs. The health workers also benefit from this personalization in two ways, first they strictly consult their patients based on their preferences, and second the health worker can fetch any information from the personalized system. Patients can get information about the treatment, background information about their conditions (the cause, symptoms, its consequences and so on) and alternative treatment and its effect.

In most of the previous personalized eHealth, the interaction between the system and the patient was fixed and simple [1]. Personalized email or text messages have also been used. This type of personalization does not consider individual preferences and interests. Suppose a centralized HIV/AIDS prevention and controlling office sends an instant message to all HIV/AID patients to take their medicine on a specified regular time; this can be considered as a group based personalized service delivery system, however this system does not address the individual preference rather it implies one size fits all notion.

Service personalization in eHealth will help to design a personalized user interface based on user's abilities, interests and needs. This will create an opportunity in addressing 
heterogeneous preferences of individual users. For instance, for an illiterate mobile user, it is possible to create a voice based user interface (interactive dialog) that can be accessed by pressing a single key.

In our framework, the user can request his preference based on priority. We apply MoSCoW (Must have, Should have, Could have and Won't have) list based preference selection. For each of the preference requirements we assigned values $1,0.75,0.5,0$ respectively. This values helps to calculate the similarity between the requested and advertised services and to rank the candidate services.

\section{USER PROFILE}

Service discovery is a mechanism for finding services. Current mechanisms work mostly semantically and syntactically by matching service requests and service offerings based on keywords and meanings or semantics. This often leads to poor result sets as the ambiguity of keywords may result in mismatches between the user query and service descriptions.

User profile or personalization of service (hereafter we use user profile or personalization interchangeably) is rarely used in service orientation; therefore we propose user profile (personalization) to be incorporated as one of the main components of service discovery in addition to contextawareness and semantics. A user profile may contain many individual data items (information, preference and rules) coming from different sources ([2], [6], [4]). When a user wishes to have the behavior of services to be personalized to his/her requirements a profile will be required [4]. Some of the values of these items will either not change (static) or change very irregularly and (dynamic) and some may be individually changed by the user.

The main purpose of user profile is to keep track of the user related information to control its availability and facilitate service access for its users, and to realize a better user experience. Besides personalization helps to identify service delivery mechanism (text, audio, video, etc.), to choose the type of service to be delivered with respect to user preferences (physical, psychological, environmental, and so on) and to create a global personalization scheme [4].

User profile determines the method of service delivery. For example, vision impaired persons wish to get the service in audio while hearing impaired users wish to receive it in text rather than audio ([2], [6], [4]).

User profiles are required to be prioritized to overcome conflicting profiles. A user may have several preferences, however, services might not be available for the preferences to match. The priority of user preferences will help to relax the matchmaking process. In line to this ([6], [4]) stated eHealth profiles to be allocated higher priorities than non-eHealth profiles. eHealth profiles are considered as more important than personal profiles (normal profiles) as they contain information (extracted from electronic health records) and preferences ([2], [6], [4]).

Generally, the user profile should address the following questions: where a user is located, what her job/ profession is, what her preferences (interest) is, what the context of the user is, who the provider of the service is, the cost and availability of the service ([6], [4]).

\section{A. Challenges of user profile (personalization)}

However, personalization (personal profile) is not without the challenges; some of the challenges are: how can personal information be gathered, how is this information stored in a persistent and ubiquitous manner (this is applicable for infrastructure-less networks), how can the changes always be reflected in the services rendering process? In addition, privacy is the other bottleneck in personalization. In regard to gathering information, we propose pull and push model as well as manual and automatic information gathering. Manually, the user will fill in the form and continuously update the profile whenever there is a change whereas in automatic information gathering especially, in mobile ad hoc networks, every node can broadcast its profile to the immediate neighbor node and gathering information from profile providers and devices such as sensors, GPS, etc. In the case of user profile privacy, in our framework, we proposed user authentication and authorization model to secure the user profile from privacy threat (see section 2.3).

\section{B. Working example}

John works in non governmental charity organization. After a few days in a charity work he feels a little discomfort. He has fever, headache and nausea. The village health extension worker (HEW) diagnoses a laboratory examination (blood, stool, etc), however, all the results are negative. The HEW makes additional examination to find the cause to administer the appropriate treatment. But the case of John cannot be resolved in the health post as there is only one small laboratory and there is no specialist. Thus the HEW decides to access the health system to get additional help. After logging into the system, the HEW fills the form, entering the diagnosis made, the administered medication, the patient's symptoms, the patient's history, John's preferences and the location of the hospital. After accessing John's medical history (Electronic Health Record) remotely, the HEW learns that John has a mild hypertension case and is allergic for antibiotics, and John prefers not to have an injection. Based on this information, some services are retrieved from the repository. After the system receives the information, it forwards all this information to John's family doctor (if any) and the emergency dispatch center (Emergency dispatch center is a specialized or referral hospital near by the given location area. This dispatch centers which has good medical facilities and a number of specialists). While receiving this emergency request the system will 
forward the cases to the appropriate specialist to assist the patient remotely. These emergency team will communicate the patient's private doctor and forward to the attending doctor the treatment to be administered for the patient.

As shown in Figure 2 a user may use two ways of requesting a service: (1) using voice and (2) using text or/and video (depending on the bandwidth in developing countries context). As most of the population in developing countries are illiterate, especially in the rural areas, voice based communication will increase the number of eHealth system users. Furthermore, users who own a smart phones and can use the features of the smart phone or PDA, can query service using text and video (if the infrastructure allows). As depicted in the Figure 2, the user can press a button and send a voice request. The voice server (VoiceXML) converts the voice data into text data, and forward the converted request to the application server. After receiving the request, the application server will forward the request to the appropriate emergency dispatch centers (EDC) based on the location. EDC is a center which is set up based on the geographical location. This center should have a number of specialist doctors, good health facilities and services. After evaluating the user request, the EDC forwards the request to the appropriate specialists. Finally, the specialists evaluate the user request, verify and access the patient's history (EHR), communicate with the family doctor (if any) of the patient, and access the service repository. Finally, the treatment and its procedure is forwarded to the mobile of the requesting HEW.

The most prominent benefits of voiced based questionanswering dialog using mobile devices (mobile, PDA,...) are:

- it enables the underprivileged to create, host and share information and services produced by themselves,

- it provides simple and affordable access mechanisms to let the masses exploit IT services and applications similar to the ones that are currently available to WWW users, and,

- it provides a cost effective ecosystem that can be made available over the infrastructure that exists today to create and sustain a community parallel to the WWW.

A voice based querying services has its own challenges. But in the case of Ethiopia it is very important since the majority of the population does not speak English rather can they use their own language. Choosing the language to be localized is another challenge since many languages are spoken in Ethiopia. In our project we plan to use Amharic and English as a medium of communication in our system. The former is understood by many of the people and is the working language of the country and the latter is the medium of instruction starting form junior high schools, high schools and higher education institutions.

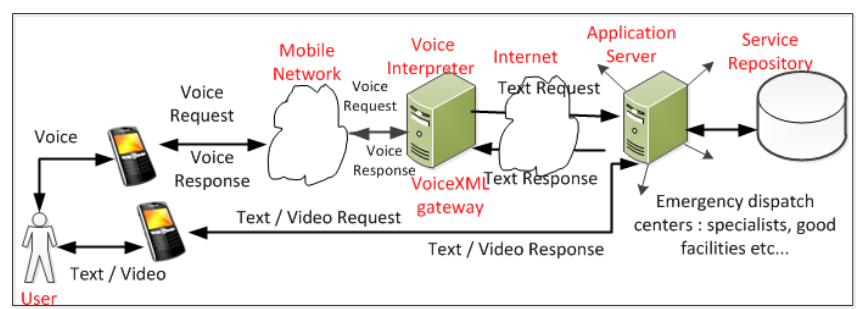

Figure 2. Personalized user service request

\section{CONCLUSION}

In this paper we try to address the issue of personalized service discovery for the healthcare domain. The main importance of personalization is to address the users' request based on their preferences and interests. Above all, personalization helps to develop the user interface for the users on the basis of their literacy level and affiliations. In our framework we propose a voice based requesting services for rural users and community health workers using their local languages. As a future work we will employ the proposed framework using mobile phone in Amharic language. There will be some limitations to use Amharic has very limited Automatic speech recognition resources.

\section{REFERENCES}

[1] A. Cawsey, F. Grasso, and C. Paris. Adaptive information for consumers of healthcare. Springer-Verlag Berlin Heidelberg, May 2007.

[2] O. Choi and S. Han. Personalization of rule-based web services. Sensors, 8(4):2424-2435, 2008.

[3] C. DiMarco, D. Wiljer, and E. Hovy. Self-managed access to personalized healthcare through automated generation of tailored health educational materials from electronic health records. In Virtual Healthcare Interaction: Papers from the AAAI Fall Symposium (FS-09-07,, AAAI Fall Symposium Series), pages 4-7, 2009.

[4] ETSI. Human factors (hf); user profile management. In ETSI EG 202325 V1.1.1, ETSI Guide, pages 1-100, 2005.

[5] Hl V Methodology, Hl The Organization, and Hl The Organization. H17 organizational overview.

[6] F. Petersen, M. Bartolomeo, G. andPluke, and T. Kovacikova. Context sensitive personalization utilizing user profiles: Standardization work at european telecommunications standards institute (etsi. In ETSI, Second International Conference on Advances in Human-Oriented and Personalized Mechanisms, Technologies, and Services, pages 20-25, 2009.

[7] D. Zhang, Z. Yu, and X.Y. Chin. Context-aware infrastructure for personalized healthcare. The International Workshop on Personalized Health, December 13-15, Belfast, Northern Ireland, 2004. 